\title{
ON TRANSVERSALS OF SIMPLY CONNECTED REGIONS
}

\author{
ERNEST C. SCHLESINGER ${ }^{1}$
}

1. Introduction. In connection with work on the boundary behavior under conformal mapping [4] I have had to consider certain curves, called "transversals" ${ }^{2}$ below. These are generalizations of crosscuts of a simply connected region of the extended plane. Transversals are defined by the principal separation property of crosscuts; namely, they divide a simply connected region into two regions that are again simply connected. This note is devoted to proving the theorem that the union of two transversals of a simply connected region has a complement (relative to the region) all of whose components are themselves simply connected regions. Furthermore, the relative boundary of each of these regions is either a Jordan curve or a union of transversals.

Our theorem is an extension of a theorem of Kerékjártó [2, p. 87; 3, p. $168 ; 5$, p. 108] to the effect that all complementary regions of a pair of intersecting Jordan curves are simply connected and have Jordan curves for their boundaries. The proof is based on the observation that our theorem is actually a version of Kerékjártó's result, provided the latter is applied on the Alexandroff one-point compactification (see for example [1, p. 23]) of the given region.

Transversals have the desirable property that they are invariant under topological mappings of the (open) region. This is no longer true of ordinary crosscuts.

2. Definitions. We recall that a region is an open connected subset of the extended plane. A region is called simply connected in case its boundary is a continuum (which may degenerate to a point) or empty, that is, in case its complement in the extended plane is connected.

A crosscut of a region $\Omega$ is a homeomorphic image of the open interval $(0,1)$ in $\Omega$ with the property that the homeomorphism is the restriction of a continuous map of the closed interval $[0,1]$ into the closure $\bar{\Omega}$ of $\Omega$, where the images of 0 and 1 lie on $\Gamma=$ bd $\Omega$. (We do not exclude the possibility that these two "endpoints" of the crosscut

Presented to the Society, October 28, 1961; received by the editors May 9, 1961.

1 The author acknowledges with gratitude several clarifying and stimulating conversations with Professors G. P. Johnson and H. Tong.

2 These curves were actually called "crosscuts" in [4]. The theorem of the present paper enables one to prove Theorem 4 of [4] without the intervention of "generalized crosscuts" - certain unions of transversals. 
coincide.) A crosscut of a simply connected region $\Omega$ separates $\Omega$ into two simply connected regions (see, for example, $[2$, p. 106; 5 , p. 110]).

A transversal $\boldsymbol{\gamma}$ of a simply connected region $\Omega$ is a homeomorphic image of the open unit interval $(0,1)$ in $\Omega$ with the property that the set-theoretic difference $\Omega-\gamma$ has exactly two components and that these are simply connected. Thus, every crosscut of a simply connected region is a transversal of the region. The converse of this statement is false, since a transversal need not have "endpoints."

Let $\Omega$ be a region. We denote its one-point compactification by $\Omega^{*}: \Omega^{*}=\Omega \cup\left(p_{\infty}\right)$, where " $p_{\infty}$ " designates the added compactifying point. If $\gamma$ is a subset of the region $\Omega$ we shall use $\bar{\gamma}$ and $\gamma^{*}$ for the closures of $\gamma$ in the extended plane and in the compactification $\Omega^{*}$, respectively.

3. The Theorem. We state Kerékjártó's theorem and give two auxiliary propositions that are needed to establish the main result.

KeréKJÁRTó's TheOREM. Let $J_{1}$ and $J_{2}$ be Jordan curves of the extended plane, and let $J=J_{1} \cup J_{2}$. If $J_{1}$ and $J_{2}$ have more than one point in common, the boundary of each component of the complement (with respect to the extended plane) of $J$ is itself a Jordan curve.

LEMMA. Let $\Omega$ be a region and let $\Gamma$ be its boundary (relative to the extended plane). If $\gamma$ is a subset of $\Omega$ then $\bar{\gamma}$ intersects $\Gamma$ if and only if $p_{\infty} \in \gamma^{*}$.

Proof. By definition, $p_{\infty} \in \gamma^{*}$ if and only if $\gamma$ meets the complement of every compact subset of $\Omega$. Clearly, this is equivalent with the condition $\bar{\gamma} \cap \Gamma \neq \varnothing$.

COROLLARY. If $\gamma$ is a transversal of a simply connected region $\Omega$ then $\gamma^{*}$ is homeomorphic to a circumference. The homeomorphism can be realized as an extension to $[0,1]$ of the homeomorphism $\phi_{0}$ of $I^{0}=(0,1)$ to $\gamma$, with the images of 0 and of 1 identified at $p_{\infty}$.

PRoof. It follows from the separating properties of transversals and from the lemma that $p_{\infty} \in \gamma^{*}$. We now consider a sequence $\left\{p_{j}\right\}$ of points $p_{j}=\phi_{0}\left(t_{j}\right)\left(t_{j} \in I^{0} ; j=1,2, \cdots\right)$ of $\gamma \subset \Omega^{*}$. The corresponding sequence of numbers $\left\{t_{j}\right\}$ has a convergent subsequence. We assume for convenience that $\left\{t_{j}\right\}$ itself converges. If $\lim t_{j} \in I^{0}$ then $p_{0}=\lim p_{j} \in \gamma$, since $\phi_{0}$ is a homeomorphism. On the other hand, if $\lim t_{j}$ is 0 or 1 then $\left\{p_{j}\right\}$ must, at any rate have a subsequence $\left\{p_{j_{k}}\right\}$ that converges to a point of the closed compact set $\gamma^{*}$. This limit point can only be $p_{\infty}$ : otherwise $\bar{\gamma}$ would have an endpoint in the 
region $\Omega$, and this would contradict the separating properties of $\gamma$. Hence, every sequence $\left\{t_{j}\right\} \subset I^{0}$ with $\lim t_{j}=0$ or $\lim t_{j}=1$ must satisfy $\lim \phi_{0}\left(t_{j}\right)=p_{\infty}$. The homeomorphism $\phi$ defined by $\phi(t)=\phi_{0}(t)$ for $t \in I^{0}$, and $\phi(0)=\phi(1)=p_{\infty}$ is the required extension of $\phi_{0}$.

THEOREM. Let $\gamma$ and $\delta$ be transversals of a simply connected region $\Omega$ whose boundary $\Gamma$ is nonempty. Then the boundary relative to $\Omega$ of any component of $\Omega-(\gamma \cup \delta)$ is one of the following: (i) a Jordan curve, (ii) a transversal of $\Omega$, or (iii) a pair of transversals. In cases (i) and (ii) this relative boundary is a subset of $\gamma \cup \delta$, while it actually coincides with $\gamma \cup \delta$ in case (iii).

Proof. We pass to the one-point compactification $\Omega^{*}=\Omega \cup\left(p_{\infty}\right)$. In view of the lemma, the transversals $\gamma$ and $\delta$ are "compactified" as $\gamma^{*}$ and $\delta^{*}$, where $p_{\infty}$ belongs to $\gamma^{*} \cap \delta^{*}$. By the corollary, $\gamma^{*}$ and $\delta^{*}$ are Jordan curves in $\Omega^{*}$.

a. Suppose that the intersection $\gamma^{*} \cap \delta^{*}$ contains at least one point besides $p_{\infty}$. Since $\Omega^{*}$ is homeomorphic to a sphere, we conclude from Kerékjártó's theorem that every component of $\Omega^{*}-\left(\delta^{*} \cup \gamma^{*}\right)$ is a Jordan region. Returning to $\Omega$ we see that every component of $\Omega-(\gamma \cup \delta)$ is a simply connected region whose boundary relative to $\Omega$ either is a Jordan curve or consists of a transversal of $\Omega$. The former possibility takes place if the boundary of the image on $\Omega^{*}$ does not pass through $p_{\infty}$, while the latter situation holds if $p_{\infty}$ does belong to the boundary of that image. Thus, we have either case (i) or case (ii).

b. On the other hand, if $\gamma^{*} \cap \delta^{*}=\left(p_{\infty}\right)$ then on returning to $\Omega$ there will be one residual region of $\Omega-(\gamma \cup \delta)$ that is a quadrangle. Its boundary will consist of $\gamma$, a connected subset of $\Gamma, \delta$, and a second connected subset of $\Gamma$. Thus, case (iii) can occur. However, for a given pair $\gamma, \delta$, there is at most one residual region of this type, and the other two are then of type (ii).

\section{Bibliography}

1. L. V. Ahlfors and L. Sario, Riemann surfaces, Princeton Univ. Press, Princeton, N. J., 1960.

2. B. v. Kerékjárt6, Vorlesungen über Topologie. I, Berlin, 1923.

3. M. H. A. Newman, Elements of the topology of plane sets of points, 2nd. ed., Cambridge, 1954.

4. E. C. Schlesinger, Conformal invariants and prime ends, Amer. J. Math. 80 (1958), 83-102.

5. G. T. Whyburn, Analytic topology, Amer. Math. Soc. Colloq. Publ. vol. 28, Amer. Math. Soc., New York, 1942.

WESLEYAN UNIVERSITY 